\title{
Contextos metodológicos para planificar clases en las ciencias económicas, administrativas y contables
}

\section{Methodological conditions for planning classes in the economic, administrative and accounting sciences}

José Fabián Fonseca Vásconez. ${ }^{1}$, Paulina Renata Arellano Garces. ${ }^{2}$, Roberto Carlos Guevara Villavicencio. ${ }^{3}$, Sandro Oscar Muela Proaño. ${ }^{4} \&$ Andrea del Pilar Ramírez Casco. ${ }^{5}$

Recibido: 10-03-2019 / Revisado: 25-04-2019 /Aceptado: 04-05-2019/ Publicado: 05-06-2019

\begin{abstract}
.
DOI: https://doi.org/10.33262/cienciadigital.v3i2.6.562

In Ecuadorian education, the Higher Education Quality Assurance Council promotes quality in order to evaluate and accredit universities and their careers, in this area various limitations have been identified in the teaching-learning process in the different Ecuadorian universities during the various evaluation processes, however, the planning process stands out as one of the main shortcomings. For this reason, it was formulated as a problem How to perfect the class planning process in the education of economic, administrative and accounting sciences? The objective was to analyze the planning process of the class in higher education from the preparatory activity of the teacher for its preparation. It was considered that the nonexistent pedagogical training of teachers from the undergraduate level is closely related to the limitations detected. Several methodological premises were presented that were presented in activities with the university professors in the different teaching units. The aforementioned premises were considered necessary to perfect the planning process of classes in higher education.
\end{abstract}

1 Universidad Técnica Estatal de Quevedo, Facultad de Ciencias empresariales, Quevedo, Ecuador, jfonsecav@uteq.edu.ec

2 Instituto Superior Tecnológico Rumiñahui, Carrera Administración, Cantón Rumiñahui, Ecuador, paurenata_2411@hotmail.com

3 Instituto Superior Tecnológico Pelileo, Carrera Contabilidad, Cantón Pelileo, Ecuador, roberto.guevara18d03@gmail.com

4 Universidad Tecnológica Indoamérica, Facultad de Ciencias Administrativas y Económicas, Ambato, Ecuador, sandromuela@gmail.com

5 Escuela Superior Politécnica de Chimborazo, Facultad de Administración de empresas, Riobamba, Ecuador, andreitapili@yahoo.es 
Keywords: Class planning, methodological premises, higher education, economic, administrative and accounting sciences.

\section{Resumen.}

En la educación ecuatoriana, el Consejo de Aseguramiento de la Calidad de la Educación Superior, promueve la calidad a efectos de evaluar y acreditar a las universidades y sus carreras, en este ámbito diversas limitaciones se han identificado en el proceso de enseñanza aprendizaje en las distintas universidades del Ecuador durante los diversos procesos de valoración, sin embargo, se destaca como una de las principales falencias el proceso de planificación. Por esta razón se formuló como problema ¿Cómo perfeccionar el proceso de planificación de la clase en la educación de las ciencias económicas, administrativas y contables? Se presentó como objetivo analizar el proceso de planificación de la clase en la educación superior desde la actividad preparatoria del docente para su confección. Se consideró que la inexistente formación pedagógica de los docentes desde el pregrado guarda estrecha relación con las limitaciones detectadas. Se plantearon varias premisas metodológicas que fueron presentadas en actividades con los profesores universitarios en las diferentes unidades docentes. Las premisas aludidas se consideraron necesarias para perfeccionar el proceso de planificación de las clases en la educación superior.

Palabras claves: Planificación de clases, premisas metodológicas, educación superior, ciencias económicas, administrativas y contables.

\section{Introducción}

El nivel de vida y progreso de un país está ligada a la cobertura, calidad y pertinencia de la formación superior que brinda a sus ciudadanos, y a la inversión que realiza en ciencia, tecnología e innovación. La Educación Superior y la investigación asociada a ella deben concebirse como un bien público en tanto su desarrollo beneficia a la sociedad en su conjunto más allá de su usufructo individual o privado.

Por tanto, debe ser una prioridad aumentar el nivel educativo, garantizando la igualdad de oportunidades para todos en el campo de la Educación Superior, la cual se ha caracterizado por ser reproductor y no transformador, en sentido progresista de la estructura de enseñanza aprendizaje al momento de impartir clases.

En este ámbito, el proceso de universalización de la educación superior en Ecuador cobra una importancia trascendental en los propósitos de lograr mayor justicia social a partir de la producción, aplicación y difusión de la cultura para todos en correspondencia con el desarrollo de los procesos sustantivos de la universidad y su impacto en la sociedad. 
Es por ello que el perfeccionamiento del pregrado en la formación de los futuros egresados de las ciencias económicas, administrativas y contables se configura como un campo de las ciencias de la educación que necesita ser atendido de manera integral por parte de los profesionales que participan en su desarrollo. En este sentido, la actividad docente en las instituciones de educación superior, de los fundamentos de la Didáctica de la educación superior y por otro, de las orientaciones metodológicas de las disciplinas, así como del trabajo metodológico.

Justamente en este proceso se desarrollan las clases como una de las formas de organización fundamentales de las diferentes asignaturas, las cuales deben ser planificadas y ejecutadas con el rigor de la Didáctica como ciencia que direcciona científicamente el proceso docente educativo.

No obstante, se han podido observar limitaciones metodológicas en los docentes de las instituciones de educación superior evaluadas durante el proceso de acreditación universitaria, análisis que se efectuó durante el desarrollo de las clases en varias asignaturas, a través de los controles realizados. Esto evidencia una inadecuada planificación de las clases, determinado en que no existe una visión didáctica, metodológica e integral por parte de los docentes que permita analizar el proceso de planificación desde aspectos didácticos generales, hasta particulares del grupo escolar y el contexto en que se desarrolla la docencia. Por esta razón se manifiesta como problema ¿Cómo perfeccionar el proceso de planificación de la clase en las ciencias económicas, administrativas y contables?

En correspondencia, se ha propuesto como objetivo: Analizar el proceso de planificación de la clase en las ciencias económicas, administrativas y contables desde la actividad preparatoria del docente para su confección. Siendo consecuentes que conocimiento en una clase es un proceso de formación, crítico, dinámico, participativo y creativo, orientado a generar experiencias de aprendizaje que produzcan una aproximación entre el conocimiento, la realidad y la producción de significados del sujeto educativo, desarrollando una serie de saberes y competencias que van incidiendo sobre su identidad personal, profesional y ciudadana, en el marco de un contexto productivo, político, social, ambiental y cultural determinado, propiciando su transformación.

\section{Metodología}

En consideración que la planeación estratégica permite delinear el éxito o fracaso al dictar clases cómo forjadora de conocimiento con los estudiantes, y sus ámbitos de estudio, coadyuvara a tener estudiantes, egresados y profesionales, más competentes, la metodología empleada para la valoración del problema y el planteamiento de soluciones, se fundamenta en métodos de nivel teórico, empírico y estadístico, como: análisis y síntesis, cualitativo cuantitativo, histórico-lógico, enfoque de sistema, la modelación, análisis comparativo, observación científica, revisión documental, encuestas entrevistas individuales y colectivas, estadística descriptiva. 


\section{Resultados}

En la universalización de la educación en ciencias económicas, administrativas y contables a nivel superior en el Ecuador, las clases son impartidas y planificadas por profesores que en su mayoría tienen limitada formación pedagógica, sin embargo, la mayoría posee experiencia en el ámbito profesional. No obstante, se les exige un proceso docente educativo de calidad en el que se logren los objetivos del proceso formativo de pregrado.

Aunque es una fortaleza para el propio proceso formativo de pregrado contar con profesionales vinculados y con experiencia en el ámbito profesional (en tanto que aportan al desarrollo de habilidades y competencias profesionales en los estudiantes, a través las diferentes formas organizativas del proceso docente educativo), la inexistente formación pedagógica conlleva a que el pensamiento didáctico de estos se encuentre limitado para concebir la planificación de las clases desde elementos metodológicos necesarios para preparar adecuadamente la actividad docente en una asignatura específica.

Es opinión de los autores de este estudio que en el proceso de planificación de las clases se deben tomar en cuenta algunas premisas metodológicas para estructurarlas en correspondencia con el contexto en que se desarrollan y las características particulares de la asignatura en cuestión. El orden en que se presentan no significa una lógica formal o lineal, sino dialéctica, en la que realmente alcanzan su valor metodológico mediante la sinergia que se logra.

Todo ello configura un proceso de planificación de las clases acorde con las exigencias didácticas y metodológicas actuales, cuyo efecto multiplicador promueva nuevas oportunidades a los estudiantes al generar entornos significativos de aprendizaje que acercan sus experiencias académicas al mundo profesional, donde pueden desarrollar una serie de capacidades integradas y orientadas a la acción, lo cual les permita ser capaces de resolver problemas prácticos o enfrentarse a situaciones considerando como un proceso de recogida de evidencias y de formulación de valoraciones sobre la medida y la naturaleza del progreso del estudiante.

Premisas Metodológicas para la planificación de las clases en la educación de las ciencias económicas, administrativas y contables.

1. Conocimiento de la Didáctica general, los principios, leyes y la estructuración externa e interna de las diferentes formas de organización del proceso docente - educativo así como las funciones didácticas de la clase: aunque parezca trivial la necesidad del conocimiento de la didáctica, para algunos docentes esta no es una ciencia y tienen la creencia que pueden lograr resultados sin tener que preparase en estos fundamentos, bastando solo sus conocimientos en los preceptos teóricos de la ciencias y el interés 
de los estudiantes por formarse como profesionales de tercer nivel en áreas económicas, administrativas y contables.

En este ámbito, olvidan que en el proceso de planificación de las clases deben concebir el cómo van a tratar de educar, vincular la instrucción con aspectos socioeconómicos actuales, así como la coherencia que debe existir entre cada uno de los componentes para lograr un aprendizaje desarrollador. Esto contribuiría a que la secuenciación lógica de cada paso y modo de actuación pedagógica prevista desde ese momento, tenga una función didáctica, específicamente las enunciadas desde esta ciencia.

2. Conocer el perfil profesional del futuro egresado de las ciencias económicas administrativas y contables y trabajar con el programa de la disciplina particular, la asignatura, sus orientaciones metodológicas y el plan temático o programa analítico (Dosificación de contenidos): conocer el modelo del profesional orienta al docente hacia la finalidad del proceso del pregrado.

Por tanto, en la planificación se abordan acciones dirigidas al cumplimiento de las exigencias de este modelo, que al mismo tiempo se particulariza en los programas de las disciplinas y asignaturas. Por tanto, la manera en que se piensa impartir el contenido refleja la intencionalidad del por qué tal actividad o acción y qué le aporta a la formación de ese futuro profesional.

Asimismo, la lógica interna de los programas que presentan las formas organizativas en las que se muestran los contenidos (que según el criterio que se defiende en este material, no debe ser dogmática, en correspondencia con el contexto y las orientaciones metodológicas de los programas) ayudan a este proceso, aunque no son suficientes si no se valoran estos elementos en su conjunto.

3. Caracterización psicopedagógica del grupo de estudiantes, condiciones materiales (locales, medios de enseñanza), horario de la clase y turno de clase precedente: esto particulariza la clase en el grupo de estudiantes, por tanto, se debe concebir atendiendo la personalidad de estos, sus intereses y motivaciones, ritmo de aprendizaje, así como características del grupo. Por ello las condiciones de los locales y los materiales docentes con los que se cuenta, son imprescindibles para la estructuración de la clase, así como escoger la forma organizativa de la misma más allá de la sugerencia del plan analítico. En ocasiones estas condiciones, unido a circunstancias particulares determinan la forma y el método a emplear, recordando que en la clase se debe instruir y educar. 
Además, en dependencia de las características psicopedagógicas de los estudiantes, el horario en que se imparte el turno de clase y el precedente, influye en el estado psíquico de estos y la preparación previa que tienen para participar activamente del aprendizaje de los contenidos de la asignatura. De modo que el docente debe planificar sus clases concibiendo el clima en que se desarrolla y propiciar un estado favorable para que los estudiantes sean protagonistas del proceso.

4. Concepción de sistema de clases. Estrategias curriculares y extracurriculares. Trabajo político-ideológico, formación en valores e interdisciplinariedad: es necesario que el docente tome en cuenta que la clase forma parte de un sistema en el que se van desarrollando, de conjunto con las otras asignaturas del plan de estudio, varias habilidades, se van formando competencias profesionales, adquiriendo conocimientos, por tanto la clase debe permitir una secuenciación lógica de estos elementos formativos, de manera que las actividades planificadas siguen esa lógica de las precedentes y las que serán impartidas. Siendo así, no pueden analizarse de forma aislada, sino como sistema, en el que cada clase aporta al perfil del profesional, alcanzando siempre estados superiores tanto en el aprendizaje como en la formación en valores, el desarrollo de las estrategias curriculares y extracurriculares como dimensiones que a través de ese sistema configuran el futuro profesional.

Es por ello que la actividad docente y el modo de actuación profesional pedagógico de los profesores debe dirigirse como parte de un sistema, tratando de que se manifiesten los contenidos precedentes como elementos que son base para desarrollar las habilidades y competencias, al mismo tiempo que de manera intencionada se fortalezcan los valores y la vida en democracia la cual conlleve a alcanzar el Buen Vivir. Por tanto, la interdisciplinariedad no puede convertirse en un discurso, sino en uno de los propósitos de cada clase.

5. Lenguaje científico de la asignatura y la comprensión de este por parte de los estudiantes: es importante desde el punto vista didáctico el tratamiento que se la da en la clase al lenguaje científico que se utiliza. Por un lado, este va formando un conocimiento de la ciencia acorde con los símbolos, códigos que se van generando y que son imprescindibles en la apropiación del contenido pero que también es necesario esclarecer para lograr una mejor compresión de las ciencias económicas, administrativas y contables y cómo este tiene una aplicabilidad durante su labor profesional que no puede quedar a un lado.

Es notable cómo muchos docentes no se detienen a explicar el significado de este lenguaje y cómo en ocasiones, determinados homomorfismos conllevan a ciertas confusiones en el proceso docente educativo. 
6. Derivación gradual de los objetivos de la clase: la comprensión de que el objetivo de la clase no emerge de la idea del profesor y que no es copiar lo que aparece en el programa quizás parezca como que no aporta a lo metodológico. Pero más allá de la explicación que existe al respecto, es una práctica que debe ser observada y ejecutada en cada momento de planificación de las clases, aspecto este que no pocas veces es pasado por alto por varios profesores que imparten la docencia en la educación superior.

Estos elementos que se han considerado llamar premisas metodológicas por los autores, se sostienen de los fundamentos de la didáctica, entre ellos sus leyes y principios, que no son un propósito de enunciar y describir en este material dado a su conocimiento general en el ámbito educacional coadyubando a la formación científica y humanística deseada en las entidades de Educación Superior del Ecuador lo cual permita contribuir al desarrollo humano y científico del país.

Por lo cual, se han tratado de explicar de manera sintética en correspondencia con las actividades docentes y el proceso formativo de los futuros egresados de las ciencias económicas, administrativas y contables,

\section{Conclusiones}

- Las metodologías de enseñanza se convierten, en un instrumento poderoso a ser usado por los docentes ya que le es útil no sólo para identificar formas de ayudar a los alumnos a adquirir conocimientos y desarrollar sus habilidades, sino también porque ayuda a mejorar la práctica pedagógica y lograr su propio crecimiento profesional, teniendo siempre como eje transversal la amplia conceptualización del Buen Vivir desde el punto de vista técnico y filosófico.

- Las limitaciones metodológicas de los profesores de las ciencias económicas, administrativas y contables para planificar las clases de las diversas asignaturas del plan de estudio, guardan relación con la inexistente formación pedagógica de estos, lo cual afecta a las competencias propias que debe tener un profesional de las ciencias económicas, administrativas y contables.

- Se considera que las premisas metodológicas propuestas son elementos necesarios para la planificación de las clases en la educación superior y contribuyen a generar entornos significativos de aprendizaje los cuales permitan desarrollar una serie de capacidades integradas y orientadas a la acción, según unos resultados de aprendizaje planificados 


\section{Referencias bibliográficas.}

Arbesú, M. (2004) El sistema modular Xochimilco. Xochimilco de la Universidad Autónoma Metropolitana [en línea], [Consulta: 23 de abril de 2019]. Disponible en: http://biblioteca.xoc.uam.mx/docs/UAM_Lecturas_Basicas_I.pdf

Acosta, H; Batista, N; (2007) La formación de valores en la Nueva Universidad: El tutor y la atención personalizada. Libro digital. Material en CD de maestría en educación superior, "La educación en valores y la tutoría en la nueva universidad cubana".

Alfonso , P.(2008) "El trabajo didáctico de los colectivos de docentes: condición básica para las transformaciones curriculares en la Universidad". Ponencia a presentar Congreso Universidad. En CD La gestión de los procesos universitarios y la gestión del profesor. Maestría en Educación Superior.

Bernal, C. (2006) Metodología de la investigación: para administración, economía, humanidades y ciencias sociales . Pearson.

Cano, G. (2008) La evaluación por competencias en la educación superior. Revista de curriculum y formación de profesorado [Consulta: 23 de marzo de 2019]. Disponible en: http://www.ugr.es/ recfpro/rev123COL1.pdf ISBN: 9789567842432

Contreras, J. (1998). Enseñanza, Curriculum y profesorado: introducción critica a la didáctica. Editorial: AKAL. ISBN 9788476006795

Diaz, A. (1997) Didactica y Curriculum, Editorial Paidós SAICF, 1997, [Consulta: 23 de marzo de 2019]. Impreso en México - Printed in México, ISBN: 968-853-362

Elsa, T.\& Niurka, M.(2006) Preparación pedagógica para profesores de la nueva universidad cubana. Material digitalizado.

Fátima, A. ( 2004) Didáctica, teoría y práctica. Editorial Pueblo y Educación. Ciudad de la Habana.

Ginoris, O. (2006)Fátima Addine Fernández, Juan Turcaz Millán. Curso de Didáctica general. Iplac. 2006. Material digitalizado

Gutiérrez, R., \& Posada, R.(2004) Tendencias mundiales en educación médica. latreia [online]. 2004, vol.17, n.2 [cited 2012-11-02], pp.130-138.Available from: http://www.scielo.org.co/scielo.php?script=sci_arttext\&pid=S01 ISSN 0121-0793. Electronic Document Format (ABNT)

Horruitiner P. (2006) La Universidad Cubana. El Modelo de Formación. La Habana: Editorial Félix Varela

Hernández, J. Quiroz, R. (2010) Evaluar por competencias. [en línea] [Consulta: 22 de marzo de 2018]. Disponible en: file:// https://bit.ly/2WljocO 
Miranda, J.C. (2017) El Concepto de Currículo Oculto. Foro Universitario, No.75, Febrero, Biblioteca Pedagógica, México

Maturana, H. (2019) Emociones y lenguaje en educación y política. 9a. ed.Santiago: ISBN: 9789567802364 Dolmen. pág. 65. Universidad pedagógica Diseño curricular y calidad educativa [en línea] [Consulta: 18 de marzo de 2019]. Disponible en: https://bit.ly/2JiDilb

Valverde, J.(2012) Modelos de evaluación por competencias a través de un sistema de gestión de aprendizaje. [en línea] [Consulta: 22 de marzo de 2019].Disponible en: http://www.rieoei.org/rie60a03.pdf

Wesselink, R. (2012) Competence-Based Education. An Example from Vocational Practice. Documento presentado por la European Research Network in Vocational Education and TrainingThe Vocational Education and Training Network (vetnet) en la European Conference on Educational Research (ecer). Universidad de Amburgo, 17-20 de diciembre. Disponible en: http://edepot.wur.nl/34925. 2003. [en línea] 2012. [Consulta: 22 de marzo de 2019].

Zilberstein J. \& Silvestre M. (2005) Didáctica desarrolladora desde el Enfoque Histórico Cultural. Méjico: Ediciones CEIDE. 


\section{PARA CITAR EL ARTÍCULO INDEXADO.}

Fonseca Vásconez, J. F., Arellano Garces, P., Guevara Villavicencio, R., Muela Proaño, S., \& Ramírez Casco, A. del P. (2019). Contextos metodológicos para planificar clases en las ciencias económicas, $\begin{array}{llll}\text { administrativas y contables. Ciencia } & \text { 252-261. }\end{array}$

https://doi.org/10.33262/cienciadigital.v3i2.6.562

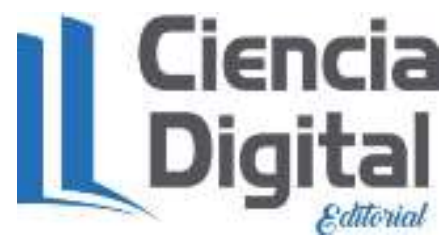

El artículo que se publica es de exclusiva responsabilidad de los autores y no necesariamente reflejan el pensamiento de la Revista Ciencia Digital.

El artículo queda en propiedad de la revista y, por tanto, su publicación parcial y/o total en otro medio tiene que ser autorizado por el director de la Revista Ciencia Digital.
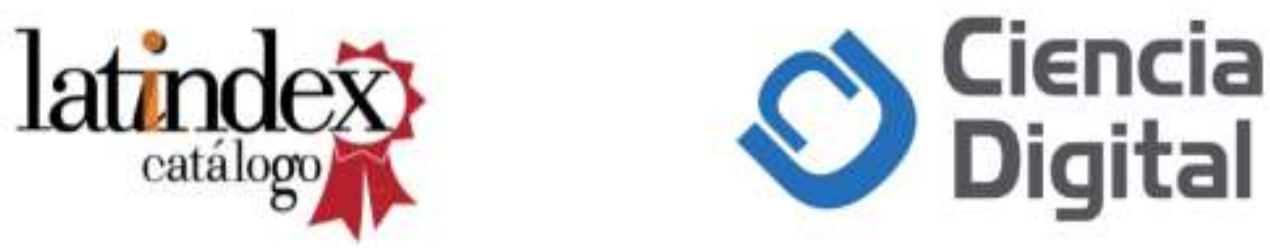